\title{
Molecular Frame Dipole Moment of Diatomic Molecules within Relativistic Coupled- Cluster Framework: A Comparative Study of Expectation Value vs. Energy Derivative Approach
}

\author{
Soumi Haldar ${ }^{1}$, Kaushik Talukdar ${ }^{1}$, Malaya K. Nayak ${ }^{2}$, Sourav Pal ${ }^{1,3}$ \\ ${ }^{I}$ Department of Chemistry, Indian Institute of Technology Bombay, Powai, Mumbai 400076, India \\ ${ }^{2}$ Theoretical Chemistry Section, Bhabha Atomic Research Centre, Trombay, Mumbai 400085, India \\ ${ }^{3}$ Indian Institute of Science Education and Research Kolkata, Mohanpur, Nadia- 741246, India
}

Email: soumihaldar@gmail.com, talukdar.kaushik7970@gmail.com, mk.nayak72@gmail.com, mknayak@barc.gov.in, spal@chem.iitb.ac.in

\begin{abstract}
:
The ground state molecular frame dipole moments of alkaline earth metal monofluorides and Group II-B-monohydrides have been computed using two analytic methods, namely the Z-vector technique and the linear expectation value method within the four-component relativistic coupled cluster singles and doubles framework. We have compared our results with the experimentally measured permanent dipole moments wherever available. It has been found that the Z-vector method, which is an energy-derivative approach predicts the molecular permanent dipole moment more accurately than the linear expectation value approach in a moderate size basis set. Further, our study shows that the high-energy virtual spinors seem to have almost no influence on the permanent dipole moment whereas the core electron correlation slightly affects this molecular property.
\end{abstract}

\section{Introduction:}

The knowledge of molecular permanent dipole moment of heavy element-molecules is one of the key requirements in the field of ultracold physics which is one of the most interesting topics of increasingly growing interest in recent years. When an ultracold molecule possesses permanent dipole moment (PDM), the applicability of them becomes larger due to the presence of long range anisotropic dipole-dipole interactions that often gives rise to some exotic and elusive quantum phases like supersolid phase ${ }^{1,2}$. Dipole interactions between polar molecules can couple the basic units of quantum information i.e. qubits ${ }^{3}$ which are described as molecular electric dipoles oriented along or against some external electric field. Another research domain where PDM of molecules play a key role is the formation of quantum liquid of dipolar chains arising from the anisotropy and the long range character of dipolar interactions ${ }^{4}$. PDM of heavy hetero-diatomic open-shell molecules has one of the greatest applicability in the experimental search of 
electron electric dipole moment (eEDM) $)^{5}$ These molecules possess a large internal effective electric field due to which the experimental sensitivity to the eEDM is enhanced hugely ${ }^{6,7}$.

Thus, accurate prediction of molecular PDM by theoretical method is essential since experimental measurement of PDM is not always feasible due the very short lifetime of the molecules. Some of the most significant progresses in this field have taken place by the theoretical determination of PDMs of alkaline earth metal halides. Torring et al. ${ }^{8}$ proposed the ionic Rittner model ${ }^{9}$ to calculate the PDMs of a few molecules of this group. A ligand field approach (LFA) was developed by Rice, Martin and Field ${ }^{10}$ for the calculation of PDMs along with other properties of calcium monohalides. In recent times, numerous wavefunction-based methods have been successfully employed to compute the molecular frame dipole moments for a number of diatoms. Usually the properties calculated by such methods demand very accurate wavefunctions and development of such methods is therefore not trivial. The first ab initio calculation of PDMs of alkaline earth metal monohalides was reported by Honjou et al. ${ }^{11}$ for $\mathrm{CaCl}$, where they employed the state-averaged multi-configuration self-consistent-field (SA-MCSCF)/configuration interaction (CI) wave function. Later, Langhoff and group ${ }^{12}$ showed that a coupled pair functional (CPF) approach is found to give considerably better estimate of dipole moments as compared to the single reference singles plus doubles configuration-interaction (SDCI) model. Kobus et al. ${ }^{13}$ compared the dipole moments of these molecules obtained from finite basis set and finite difference Hartree-Fock (FD-HF) calculation. Due to the fact that electron correlation effect is not considered within the HF domain, the accuracy of their results decreases as the molecule gets heavier. Bundgen et al. ${ }^{14}$ reported the PDM of the ground state of $\mathrm{CaF}$ obtained from multi-reference configuration-interaction (MRCI) calculation. PDMs of BeF, MgF and CaF were calculated using the second order Moller-Plesset perturbation (MP2) theory by Buckingham et al. ${ }^{15}$

All the above mentioned semi-empirical and ab initio calculations of PDM were performed in a nonrelativistic framework. However, from several previous investigations carried out by different groups ${ }^{16-}$ ${ }^{20}$ it has now been established that PDM is largely affected by the spin-orbit coupling effects along with the electron correlation in a molecule, especially for the heavy ones. Therefore, a suitable theoretical method for accurate calculation of PDM would be the one which can capture the interplay of relativistic and correlation effects correctly. The four-component Dirac-Hartree-Fock (DHF) method can incorporate the relativistic effects in the best possible way for many-electron systems within a single determinant framework. On the other hand, coupled-cluster (CC) method has earned the reputation of being one of the most efficient and reliable computational tools for the treatment of correlation effects. Thus, relativistic coupled cluster (RCC) method would be a worth choice for accurate calculation of molecular PDM. It should be noted here that molecular property within relativistic CC framework can be obtained either employing energy derivative approach or expectation value method. 
Several pioneering work in the RCC methods for the study of structure and properties of heavy systems have been developed by Visscher et al. ${ }^{21,22}$, Petrov et al. ${ }^{23}$, Ishikawa and co-workers ${ }^{24-26}$, Saue and co-workers ${ }^{27}$ and Sasmal et al. ${ }^{28}$ A fully relativistic calculation of PDMs of the alkaline earth metal monofluoride series (except RaF) was reported by Das and coworkers. ${ }^{19}$. They implemented a linearized expectation value approach within the relativistic coupled cluster singles and doubles (RCCSD) framework. Sasmal et al. ${ }^{29}$ improved upon their result for $\mathrm{SrF}$, using an energy derivative approach. They also calculated the PDM of RaF using the same method ${ }^{30}$. Theoretical investigations of the ground and lowest excited states of $\mathrm{BaF}$ and its lighter homologs were carried out in a relativistic Fock-space $\mathrm{CC}$ method by Hao et al. ${ }^{31}$ Most recently Bala et al. ${ }^{20}$ have reported the PDMs of alkaline earth metal monofluorides (AEMF) at the Kramers-restricted CI level of theory limited to the single and double excitations (KRCISD).

A large number of the above-mentioned relativistic calculations of PDMs have been performed using the expectation value approach ${ }^{16-19,32}$. Now this expectation value approach within a CC theory is not very straight-forward as it suffers from difficulties arising from the presence of the de-excitation operator $T^{\dagger}$ in the expression for the expectation-value CC property:

$\bar{O}=\frac{\left\langle 0\left|e^{\hat{T}^{\dagger}} \hat{O} e^{\hat{T}}\right| 0\right\rangle}{\left\langle 0\left|e^{\hat{T}^{\dagger}} e^{\hat{T}}\right| 0\right\rangle}$,

where $|0\rangle$ is the reference state, $\hat{O}$ is the property operator, and, $\hat{T}$ and $\hat{T}^{\dagger}$ are the excitation and deexcitation operator respectively.

This series becomes non-terminating in an attempt to factorize the numerator so that a factor of it gets canceled out by the denominator. In order to obtain practical working equations, the series needs to be truncated. Thus, the calculation of expectation value in CC theory is not a trivial one and several truncation schemes have been proposed in literature to overcome this issue ${ }^{33,34}$. An elegant truncation hierarchy of expectation value CC method was developed by the Warsaw group ${ }^{33}$. They introduced a new operator that satisfies a linear CC type equation involving a finite commutator series involving the cluster operator. This method has been successfully used to calculate one-electron properties of molecules ${ }^{35,36}$. The expectation value approach adopted by Prasanna et al ${ }^{17-19,32}$, to calculate the PDM of heavy elements and their molecules, on the other hand, uses an ad hoc scheme where only the linear terms of the CC wave-function have been taken since they provide the most dominant contributions ${ }^{37}$.

The energy derivative approach within the relativistic coupled cluster framework were also developed to compute various molecular properties. For example, Pal and co-workers ${ }^{28-30,38,39}$ implemented Z-vector method within RCCSD framework to predict molecular properties such as permanent dipole moments, hyperfine structure constants, and P,T-odd interaction constants. Due to the non-variational character of $\mathrm{CC}$ theory, it does not hold the Hellmann-Feynman theorem and thus there is always a 
numerical difference in the property-values obtained from the expectation-value method and the energy derivative approach, at several levels of truncated CC. Therefore, it is important to investigate how the molecular properties (especially PDM) obtained from an expectation value method approximated to only the linear terms in the $\mathrm{CC}$ wave-function (LEV) differ from those obtained from the energy derivative method such as Z-vector technique.

In this work we have employed the linear expectation value (LEV) method $^{30}$ and the $\mathrm{Z}$-vector ${ }^{29,40,41}$ method in a RCCSD framework to compute the molecular frame permanent electric dipole moment of the ground state of AEMFs and Group II-B metal monohydrides. The highly polar AEMF molecules are of huge interest in the field of laser cooling. On the other hand, the monohydrides of the II-B metals usually possess a low molecular frame dipole moment that can be helpful for the matrix-isolated non-spectroscopic solid state eEDM search experiments ${ }^{6}$. Although several theoretical investigations of PDMs of AEMFs have been reported, study of PDMs of Group II-B metal hydrides is relatively lesser. Therefore, we have performed a systematic study of PDMs of AEMFs and II-B-Hydrides using Z-vector and LEV method. An explicit analysis of the different contributing factors is discussed, and the different trends of correlation effects in accurate prediction of the PDMs are investigated.

The paper is structured as follows: In sec. II, a brief overview of the theory of molecular PDM and the methods employed in our calculations are discussed. In sec. III, the computational details are given. Sec. IV contains the results and interpretations obtained from our study and also a comparison has been drawn between the expectation value and the Z-vector approach. Finally, the concluding remarks are presented in sec. V.

\section{Theory and Methodology:}

In a heteronuclear diatomic molecule, the electron density is usually distributed unequally between the two nuclei which results in a PDM in the molecule. Within the Born-Oppenheimer approximation, the total PDM of a molecule can be expressed as a sum of electronic and nuclear contribution. In such a case, one can define the dipole moment operator $D$ as:

$D=-\sum_{i} e \vec{r}_{i}+\sum_{A} Z_{A} e \vec{r}_{A}$

where $e$ is the charge of the electron, $\vec{r}_{i}$ is the position vector of $i^{\text {th }}$ electron from the origin, $Z_{A}$ is the atomic number and $\vec{r}_{A}$ is the position vector of $A^{\text {th }}$ nucleus.

In presence of a weak, external, static and homogeneous electric field of strength $\varepsilon$, the total energy of a system in its ground state can be expressed as a Taylor series expansion: 
$E(\varepsilon)=E^{(0)}+\left(\frac{d E}{d \varepsilon}\right)_{0} \varepsilon+\frac{1}{2 !}\left(\frac{d^{2} E}{d \varepsilon^{2}}\right)_{0} \varepsilon^{2}+\ldots$

where the negative coefficient of the second term evaluated at $\varepsilon \rightarrow 0$ represents the PDM of the system.

$\mu_{0}=-\left(\frac{d E}{d \varepsilon}\right)_{0}$

The first and the second terms in Eq. (1) represent the electronic and the nuclear contributions, respectively. Electronic contribution to the total PDM depends on the electronic structure of the molecule, however, the other term depends on the nuclear charge and position vectors of the nuclei. Our present work is mainly concerned about the electronic structure dependency of the molecular PDM. It is worth mentioning here that the accurate calculation of the PDM requires an accurate wave-function especially in the region far from the nuclei.

Dirac-Hartree-Fock (DHF) method is the most preferred method in the single reference framework to take into account the relativistic effects in atoms and molecules, however, it misses out the electron correlation effects. The dynamic part of electron correlation can be efficiently included employing the single reference coupled cluster theory. Hence, in this work, we have calculated the PDMs of the molecules using the coupled-cluster singles and doubles (CCSD) wave-function, generated from the Dirac-HartreeFock determinant, as the reference.

The coupled cluster wave function $|\psi\rangle$ is obtained by operating an exponential cluster operator on a reference state $\left|\phi_{0}\right\rangle$,

$|\psi\rangle=e^{T}\left|\phi_{0}\right\rangle$

As mentioned above, we choose the reference state $\left|\phi_{0}\right\rangle$ to be the DHF ground state wave function built from the occupied single particle 4-component Dirac spinors. It is a single determinant of open shell doublet configuration for the systems considered in the present work. $T$ is the coupled-cluster excitation operator defined as the summation of all singles to $N^{\text {th }}$ order excitation operators

$T=T_{1}+T_{2}+\ldots+T_{N}=\sum_{n}^{N} T_{n}$

with 
$T_{m}=\frac{1}{(m !)^{2}} \sum_{\substack{i j \ldots \ldots \\ a b \ldots}} t_{i j \ldots \ldots}^{a b \ldots} a_{a}^{\dagger} a_{b}^{\dagger} \ldots a_{j} a_{i}$

The occupied and unoccupied spinors are denoted as $i, j \ldots$ and $a, b \ldots$ respectively. $a_{p}^{\dagger}$ and $a_{p}$ are the creation and annihilation operators respectively corresponding to the $p^{\text {th }}$ spinor, and $t_{i j . . .}^{a b . .}$ are the cluster amplitudes. Within CCSD framework, $T=T_{1}+T_{2}$. The unknown cluster amplitudes for CCSD wavefunction are solved by the following equations

$$
\begin{aligned}
& \left\langle\phi_{i}^{a}\left|\left(H_{N} e^{T}\right)_{c}\right| \phi_{0}\right\rangle=0 \\
& \left\langle\phi_{i j}^{a b}\left|\left(H_{N} e^{T}\right)_{c}\right| \phi_{0}\right\rangle=0
\end{aligned}
$$

where, $H_{N}$ is the normal-ordered Hamiltonian. The subscript $c$ denotes the connectedness of the diagram which ensures the size extensivity of the coupled-cluster method. Throughout in this work, we have chosen the four-component Dirac-Coulomb (DC) Hamiltonian to incorporate the relativistic effects in our calculations, where the DC Hamiltonian is defined as

$$
H_{D C}=\sum_{j}\left[-i c(\vec{\alpha} \cdot \vec{\nabla})_{j}+\left(\beta-I_{4}\right) c^{2}+V^{n u c}\left(r_{j}\right)+\sum_{k>j} \frac{1}{r_{j k}} I_{4}\right] .
$$

Here $\alpha$ and $\beta$ are Dirac matrices, $c$ is the speed of light, $I_{4}$ is a $4 \times 4$ identity matrix and $i$ is electron index. $V^{n u c}\left(r_{i}\right)$ is the potential function for a finite size nucleus with Gaussian charge distribution ${ }^{42}$. The correlation energy can be calculated by the following equation

$$
E_{\text {corr }}=\left\langle\phi_{0}\left|\left(H_{N} e^{T}\right)_{c}\right| \phi_{0}\right\rangle
$$

Once the coupled-cluster wavefunction is solved, one can calculate the molecular properties using either numerical method such as finite field method or analytic approach. Here in this work, we adopt two analytic approaches, viz; expectation value and energy-derivative method to compute the molecular PDMs.

In the expectation value method the PDM $d$ is expressed as the expectation value of the electric dipole operator $D$ with respect to the molecular ground state wave function $\psi$. 
$d=\frac{\langle\psi|D| \psi\rangle}{\langle\psi \mid \psi\rangle}$

It was shown that the denominator cancels the unlinked part of the numerator leading to a fully connected series involving the numerator ${ }^{43}$.

Thus, in the RCC framework,

$$
\begin{aligned}
& d=\left\langle\phi_{0}\left|e^{T^{\dagger}} D_{N} e^{T}\right| \phi_{0}\right\rangle_{c}+\left\langle\phi_{0}|D| \phi_{0}\right\rangle \\
& =\left\langle\phi_{0}\left|e^{T^{\dagger}} D_{N} e^{T}\right| \phi_{0}\right\rangle_{c}+\left\langle\phi_{0}\left|\left(-\sum_{i} e r_{i}\right)\right| \phi_{0}\right\rangle+\sum_{A} Z_{A} e r_{A}
\end{aligned}
$$

The terms in the above expression represent the electron correlation effects $\left(\mathrm{EC}_{\text {corr }}\right)$, electronic contribution from Dirac-Hartree-Fock (DHF) level $\left(\mathrm{EC}_{\mathrm{DHF}}\right)$, and nuclear contribution (NC) respectively. The first two terms as a whole represent the electronic contribution (EC). The term corresponding to the correlation contribution is a non-terminating series and therefore needs to be truncated forcefully. Since the leading contributions come from the linear terms in $\mathrm{CC}$ wave-function, an approximation is made by taking only the linear terms in the CCSD wave function ${ }^{37}$.

$$
\left\langle\phi_{0}\left|e^{T^{\dagger}} D_{N} e^{T}\right| \phi_{0}\right\rangle_{c}=\left\langle\phi_{0}\left|\left(1+T_{1}^{+}+T_{2}^{+}\right) D_{N}\left(1+T_{1}+T_{2}\right)\right| \phi_{0}\right\rangle_{c}
$$

Z-vector method is based on an energy derivative approach, ${ }^{40,41,44}$ where the molecular property is determined as the derivative of energy with respect to a suitable external perturbation.

In the Z-Vector method the derivative terms associated with the cluster amplitudes are included by introducing a perturbation independent linear operator $\Lambda^{41}$. The antisymmetrized operator $\Lambda$ is defined as a summation of all singles to $N^{\text {th }}$ order de-excitation operators.

$$
\Lambda=\Lambda_{1}+\Lambda_{2}+\ldots+\Lambda_{N}=\sum_{n}^{N} \Lambda_{n}
$$

where

$$
\Lambda_{m}=\frac{1}{(m !)^{2}} \sum_{\substack{i j \ldots \ldots \\ a b \ldots}} \lambda_{a b \ldots . . .}^{i j \ldots} a_{i}^{\dagger} a_{j}^{\dagger} \ldots a_{b} a_{a}
$$

and $\lambda_{a b \ldots}^{i j \ldots}$ are the amplitudes corresponding to the operator $\Lambda_{m}$. Within the CCSD framework $\Lambda=\Lambda_{1}+\Lambda_{2}$ , and the amplitude equations for $\Lambda_{1}$ and $\Lambda_{2}$ are given by 


$$
\begin{aligned}
& \left\langle\phi_{0}\left|\left[\Lambda\left(H_{N} e^{T}\right)_{c}\right]_{c}\right| \phi_{i}^{a}\right\rangle+\left\langle\phi_{0}\left|\left(H_{N} e^{T}\right)_{c}\right| \phi_{i}^{a}\right\rangle=0 \\
& \left\langle\phi_{0}\left|\left[\Lambda\left(H_{N} e^{T}\right)_{c}\right]_{c}\right| \phi_{i j}^{a b}\right\rangle+\left\langle\phi_{0}\left|\left(H_{N} e^{T}\right)_{c}\right| \phi_{i j}^{a b}\right\rangle+\left\langle\phi_{0}\left|\left(H_{N} e^{T}\right)_{c}\right| \phi_{i}^{a}\right\rangle\left\langle\phi_{i}^{a}|\Lambda| \phi_{i j}^{a b}\right\rangle=0 .
\end{aligned}
$$

Once the amplitudes are known, the molecular permanent dipole moment can be derived as the first order energy derivative with respect to the external electric field $\varepsilon$ which is given by:

$$
d=-\left(\frac{d E(\varepsilon)}{d \varepsilon}\right)_{\varepsilon=0}=-\left(\left\langle\phi_{0}\left|\left(O_{N} e^{T}\right)_{c}\right| \phi_{0}\right\rangle+\left\langle\phi_{0}\left|\left[\Lambda\left(O_{N} e^{T}\right)_{c}\right]_{c}\right| \phi_{0}\right\rangle\right)
$$

Here $O_{N}$ is the derivative of the normal-ordered perturbed Hamiltonian with respect to the external field of perturbation.

Z-vector method, being an energy-derivative method, is a naturally terminating series at any level of CC approximation. The Z-vector method can also be seen to be identical to the constrained variational approach $^{45}$ developed by Arhus group and bears similarity to the approximate versions of linearized extended coupled-cluster approach ${ }^{46,47}$. Since the normal CC (NCC) method is non-variational in nature, generalized Hellmann Feynman theorem does not hold for NCC and thus expectation value method and energy-derivative method within the NCC framework are not the same. In fact, energy-derivative method includes some additional terms which are missing in the corresponding truncated expectation value ${ }^{41}$. Therefore, any property value obtained from energy-derivative method is expected to be much more reliable than the truncated expectation value method using only linear excitations.

In either of the two methods described above, total PDM is a result of the sum of electronic contribution (EC) and the nuclear contribution (NC). The electronic part EC consists of the (i) electronic contribution at the DHF level ( $\left.\mathrm{EC}_{\mathrm{DHF}}\right)$, which takes care of the relativistic effects in the system, and the (ii) correlation contribution at the post DHF level ( $\left.\mathrm{EC}_{\text {corr }}\right)$. So as a whole, the electronic term incorporates the interplay of different kinds of correlation and relativistic effects. While the correlation contribution to the electronic component depends on the choice of correlation method and the basis set, the electronic part coming from the DHF calculation depends only on the basis set. On the other hand, the nuclear part NC is independent of either of the correlation method and the basis set, it remains constant for a particular molecule with a particular nuclear charge, and at a particular bond length. 
$P D M=N C+E C_{D H F}+E C_{c o r r}$

It is to be mentioned here that although both the NC and EC individually depend on the choice of origin, total dipole moment remains unaffected by the choice of origin, except a change in sign of the total PDM value. However, for all the calculations in our work we have considered the metal centre as the origin.

\section{Computational Details:}

The DHF equation is solved using a locally modified version of DIRAC17 program package ${ }^{48}$. The one- and two-electron integrals along with the one-electron property integrals are also generated using this package. Finite size nuclear model with Gaussian charge distribution ${ }^{42}$ has been used and the nuclear parameters are taken to be the default values incorporated in DIRAC code. "No virtual pair approximation" $(\text { NVPA })^{49}$ is employed to discard the negative energy spectrum in our calculation. Four component DiracCoulomb (DC) Hamiltonian is used in all the calculations unless otherwise mentioned. This means, higher order relativistic term i.e. the Gaunt and Breit terms has been neglected in general. The correlation contribution of the PDM is computed using our in-house developed codes based on $\mathrm{LEV}^{30}$ and Z-vector ${ }^{29}$ methods, and are interfaced with the DIRAC17 program package. For the alkaline earth metal monofluoride series ( $\mathrm{AF}$, where $\mathrm{A}=\mathrm{Mg}, \mathrm{Ca}, \mathrm{Sr}, \mathrm{Ba}, \mathrm{Ra}$ ) three different (large component) basis sets of double-zeta (DZ), triple-zeta (TZ) and quadruple-zeta (QZ) have been used: (i) A: Dyall.cv2z ${ }^{50,51}$, F: cc-pCVDZ ${ }^{52}$ for DZ basis set, (ii) A: Dyall.cv3z ${ }^{50,51}$, F: cc-pCVTZ ${ }^{52}$ for TZ basis set, and, (iii) A: Dyall.cv4z ${ }^{50,51}$, F: cc-pCVQZ ${ }^{52}$ for QZ basis set. For the Group II-B hydrides ( $\mathrm{AH}$, where $\mathrm{A}=\mathrm{Zn}, \mathrm{Cd}, \mathrm{Hg}$ ) following basis sets have been used: (i) A: dyall.ae $2 z^{53,54}$, H: cc-pCVDZ ${ }^{52}$ for DZ basis set and (ii) A: dyall.ae $3 z^{53-55}$, H: cc-pCVTZ ${ }^{52}$ for TZ basis set . Small component basis sets are generated from the large component using the restricted kinetic balance $(\mathrm{RKB})^{56}$ condition.

Equilibrium bond lengths that have been used for the molecules are given in Table 1. All electrons are included in the correlation calculation unless otherwise mentioned. However, for the DZ and TZ basis, an energy cutoff of 500 a.u. for the virtual spinors has been used for all the molecules except RaF, for which the cutoff considered is 200 a.u. This means that all the virtual spinors having energy above 500 a.u. (200 a.u. for $\mathrm{RaF}$ ) are discarded in our calculation. $\mathrm{RaF}$ being the heaviest system among all, generates a very large number of virtual spinors with high energies. Thus, a lower cutoff has been considered for RaF for the ease of computation. Use of these cutoffs reduces the computational cost significantly. In the QZ basis, lower cutoff needs to be used at least for the heavier elements, otherwise it would be computationally too expensive. Therefore, the cutoffs used for $\mathrm{Ba}$ and $\mathrm{Ra}$ are 200 a.u. and 20 a.u. respectively in this large basis. Additionally, the 18 inner core electrons of RaF have been frozen in the QZ basis set to reduce the computation time. 
Table 1: Equilibrium bond lengths used in our calculations and electronegativity of the elements. Electronegativity data are taken from Ref ${ }^{57}$

\begin{tabular}{llll}
\hline Elements & Electronegativity & Molecule & $\begin{array}{l}\text { Bond } \\
\text { length }(\AA)\end{array}$ \\
\hline \hline $\mathrm{Mg}$ & 1.31 & $\mathrm{MgF}$ & $1.750^{58}$ \\
$\mathrm{Ca}$ & 1.00 & $\mathrm{CaF}$ & $1.967^{58}$ \\
$\mathrm{Sr}$ & 0.95 & $\mathrm{SrF}$ & $2.075^{59}$ \\
$\mathrm{Ba}$ & 0.89 & $\mathrm{BaF}$ & $2.160^{59}$ \\
$\mathrm{Ra}$ & 0.89 & $\mathrm{RaF}$ & $2.240^{60}$ \\
$\mathrm{Zn}$ & 1.65 & $\mathrm{ZnH}$ & $1.595^{61}$ \\
$\mathrm{Cd}$ & 1.69 & $\mathrm{CdH}$ & $1.780^{61}$ \\
$\mathrm{Hg}$ & 2.00 & $\mathrm{HgH}$ & $1.766^{61}$ \\
$\mathrm{H}$ & 2.20 & & \\
$\mathrm{~F}$ & 3.98 & & \\
\hline
\end{tabular}

\section{Results and Discussion:}

\section{IV.A. Alkaline Earth Metal Monofluorides:}

The total molecular frame PDMs of the monofluorides of alkaline earth metal series, obtained with DZ, TZ and QZ quality basis sets are shown in Table 2, 3, and 4, respectively. Contributions of different terms involved in the expression of the PDM are also presented in the same table.

As we can see from Tables 2, 3 and 4, all the DZ, TZ and QZ quality basis sets show similar trend in electronic contribution at the DHF level $\left(\mathrm{EC}_{\mathrm{DHF}}\right)$. A large part of $\mathrm{NC}$ gets cancelled out by the $\mathrm{EC}_{\mathrm{DHF}}$ being opposite in sign (Figure 1). Magnitude of both the $\mathrm{NC}$ and the $\mathrm{EC}_{\mathrm{DHF}}$ increases monotonically on moving from $\mathrm{MgF}$ to $\mathrm{RaF}$. This is due to the fact that the equilibrium bond length of the molecule, which is responsible for the nuclear contribution, increases as the metal gets heavier (i.e. as the $\mathrm{Z}$ increases). Also, the relativistic effect becomes more pronounced for heavier systems. It has been reported in earlier work ${ }^{20}$ that the difference in the PDM obtained from relativistic and non-relativistic calculation, increases due to the increase in relativistic effects, as one goes from lighter to heavier molecules. 
Table 2: Different contributions to the PDM and the total PDMs (T) of AEMFs with DZ basis set (PDM is presented in Debye)

\begin{tabular}{cccccccc}
\hline Molecule & NC & $\mathbf{E C}_{\text {DHF }}$ & $\mathbf{E C}_{\text {corr }}$ & $\mathbf{E C}_{\text {corr }}$ & $\mathbf{T}_{\text {DHF }}$ & $\mathbf{T}_{\mathbf{L E V}}$ & $\mathbf{T}_{\mathbf{Z} \text {-vec }}$ \\
& & & $(\mathbf{L E V})$ & $(\mathbf{Z}$-vec $)$ & & & \\
\hline \hline $\mathrm{MgF}$ & 75.65 & -78.72 & 0.32 & 0.24 & -3.07 & -2.75 & -2.83 \\
$\mathrm{CaF}$ & 85.03 & -87.80 & -0.07 & -0.13 & -2.77 & -2.84 & -2.90 \\
$\mathrm{SrF}$ & 89.70 & -92.47 & -0.19 & -0.25 & -2.77 & -2.96 & -3.02 \\
$\mathrm{BaF}$ & 93.38 & -95.71 & -0.36 & -0.42 & -2.33 & -2.69 & -2.75 \\
$\mathrm{RaF}$ & 96.77 & -100.05 & -0.26 & -0.31 & -3.28 & -3.54 & -3.59 \\
\hline
\end{tabular}

Table 3: Different contributions to the PDM and the total PDMs (T) of AEMFs with TZ basis set (PDM is presented in Debye)

\begin{tabular}{cccccccc}
\hline Molecule & $\mathbf{N C}$ & $\mathbf{E C}_{\mathbf{D H F}}$ & $\mathbf{E C}_{\text {corr }}$ & $\mathbf{E C}_{\text {corr }}$ & $\mathbf{T}_{\mathbf{D H F}}$ & $\mathbf{T}_{\mathbf{L E V}}$ & $\mathbf{T}_{\text {Z-vec }}$ \\
& & & $(\mathbf{L E V})$ & $(\mathbf{Z}$-vec $)$ & & & \\
\hline $\mathrm{MgF}$ & 75.65 & -78.70 & 0.13 & 0.06 & -3.05 & -2.92 & -2.99 \\
$\mathrm{CaF}$ & 85.03 & -87.71 & -0.27 & -0.31 & -2.68 & -2.95 & -2.99 \\
$\mathrm{SrF}$ & 89.70 & -92.43 & -0.45 & -0.48 & -2.73 & -3.18 & -3.21 \\
$\mathrm{BaF}$ & 93.38 & -95.56 & -0.71 & -0.73 & -2.18 & -2.89 & -2.91 \\
$\mathrm{RaF}$ & 96.77 & -99.86 & -0.61 & -0.63 & -3.09 & -3.70 & -3.72 \\
\hline
\end{tabular}

Although we have obtained the total PDMs as negative entities from our calculations for all the systems, we will focus only on the magnitude in our discussion. The reason behind this is that the sign convention of the electric dipole moment vector is a matter of ambiguity ${ }^{62}$ as chemists consider that the vector is directed from the positive charge towards the negative charge while according to the physicists the direction is exactly opposite. So here we are concerned only about the magnitude of the total PDM. In our calculations we have taken the alkaline earth metal atom as the origin of dipole moment. Thus, the atomic number of $\mathrm{F}$ appears in the expression of $\mathrm{NC}$ term in equation (2) for all the molecules. It is found 
in all the cases that the EC is higher in magnitude than the NC which ultimately leads to a negative sign of the total PDM.

Table 4: Different contributions to the PDM and the total PDMs (T) of AEMFs with QZ basis set (PDM is presented in Debye)

\begin{tabular}{cccccccc}
\hline Molecule & NC & $\mathbf{E C}_{\text {DHF }}$ & $\mathbf{E C}_{\text {corr }}$ & $\mathbf{E C}_{\text {corr }}$ & $\mathbf{T}_{\text {DHF }}$ & $\mathbf{T}_{\text {LEV }}$ & $\mathbf{T}_{\text {Z-vec }}$ \\
& & & $(\mathbf{L E V})$ & $(\mathbf{Z}$-vec $)$ & & & \\
\hline $\mathrm{MgF}$ & 75.65 & -78.74 & 0.04 & -0.01 & -3.09 & -3.05 & -3.10 \\
$\mathrm{CaF}$ & 85.03 & -87.73 & -0.37 & -0.41 & -2.70 & -3.07 & -3.11 \\
$\mathrm{SrF}$ & 89.70 & -92.45 & -0.59 & -0.61 & -2.75 & -3.34 & -3.36 \\
$\mathrm{BaF}$ & 93.38 & -95.63 & -0.83 & -0.83 & -2.25 & -3.08 & -3.08 \\
$\mathrm{RaF}$ & 96.77 & -99.95 & -0.66 & -0.67 & -3.18 & -3.84 & -3.85 \\
\hline
\end{tabular}

We found that the absolute value of the total PDM ( $\left.\mathrm{T}_{\mathrm{DHF}}\right)$ obtained by considering only the NC and EC at the DHF level has no proper trend for the AEMF series. However, when we incorporate the correlation contribution to the DHF values of the PDM, it is found that the total PDM obtained from either of the two correlation methods increases with increasing atomic number of the metal, with an exception for $\mathrm{BaF}$. This happens for both the methods with all three DZ, TZ and QZ standard basis sets. This may be due to the following fact: The dipole moment is determined mostly by two factors: (i) difference in electronegativity between the two bonded atoms and (ii) distance between the $\delta^{+}$and $\delta^{-}$charges (i.e. equilibrium bond length). Larger the difference in electronegativity and larger the charge separation, larger is the dipole moment. As can be seen from Table 1, the equilibrium bond length increases monotonically on going from top to bottom along the alkaline-earth-metal-monofluoride series. The electronegativity of the alkaline-earth-metals decreases monotonically from top to bottom along the group. Thus, since F is the highest electronegative atom, the difference in electronegativity between the metal and the F atom increases as one moves from $\mathrm{MgF}$ to $\mathrm{RaF}$ leading to an increase in the bond polarity. The exceptional decrease in the total PDM as we go from $\mathrm{SrF}$ to $\mathrm{BaF}$ can be interpreted by an analogous explanation given by Das and coworkers $^{19}$. If we closely examine the difference in $\mathrm{NC}$ and the difference in total electronic contribution $\mathrm{EC}\left(\mathrm{EC}_{\mathrm{DHF}}+\mathrm{EC}_{\mathrm{corr}}\right)$ between two successive molecules, we can see that in almost all the cases, the magnitude of the difference in EC is larger than that of the NC. The only exception is found for the SrF$\mathrm{BaF}$ pair, for which the difference in $\mathrm{NC}$ dominates over the difference in $\mathrm{EC}$. Being a positive parameter, 
the effect of nuclear term is to increase the PDM, while the electronic term reduces it, being negative in sign.

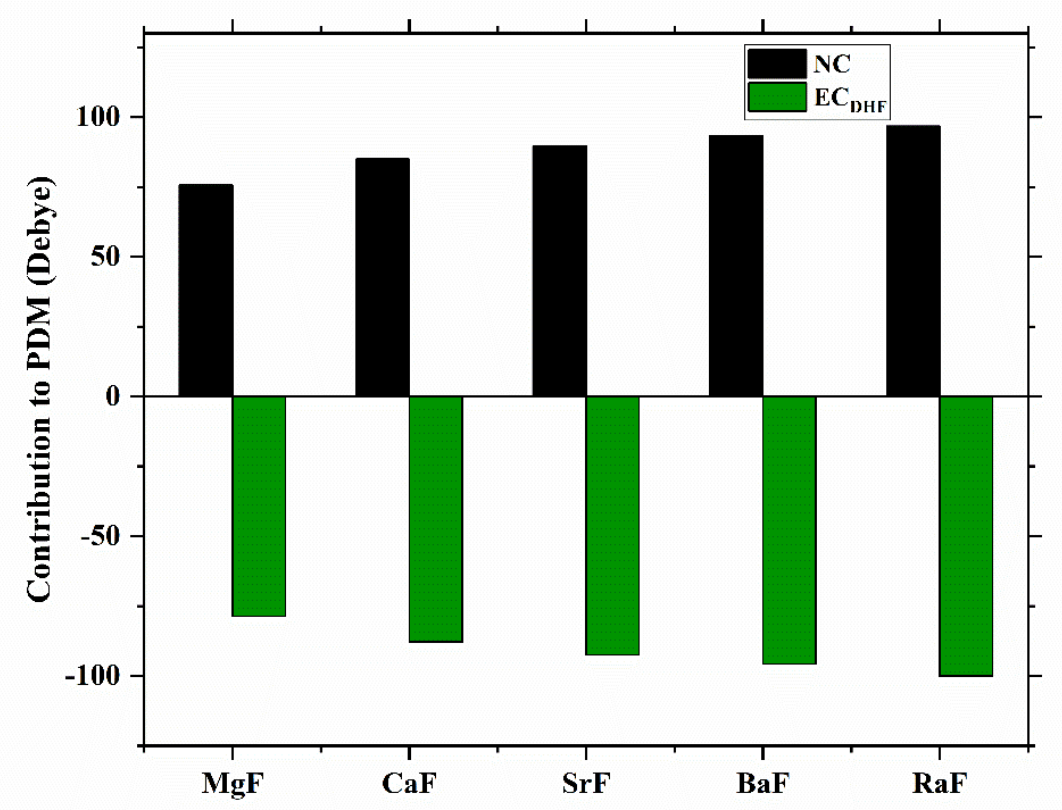

Fig 1: Nuclear contribution (NC) and electronic contribution at DHF level ( $\left.\mathrm{EC}_{\mathrm{DHF}}\right)$ (both in Debye) to the total PDMs of AEMFs. Basis: Double Zeta

Thus, the PDM of BaF becomes less negative than the other molecules in the series, since the negatively signed EC is dominated by the positively signed NC. In fact, this order of dipole moment agrees well with the experimental values which will be discussed later.

The total PDMs obtained from DHF, LEV and Z-vector calculations are presented in Figure 2. Although it is difficult to comment on the trend in the correlation effect as a separate entity, on moving through successively heavier elements, one can clearly see that a combination of four-component DHF and either of the two post-DHF correlation calculations, can predict the correct picture of the interplay between relativistic and correlation contribution to the total PDMs of molecules. 


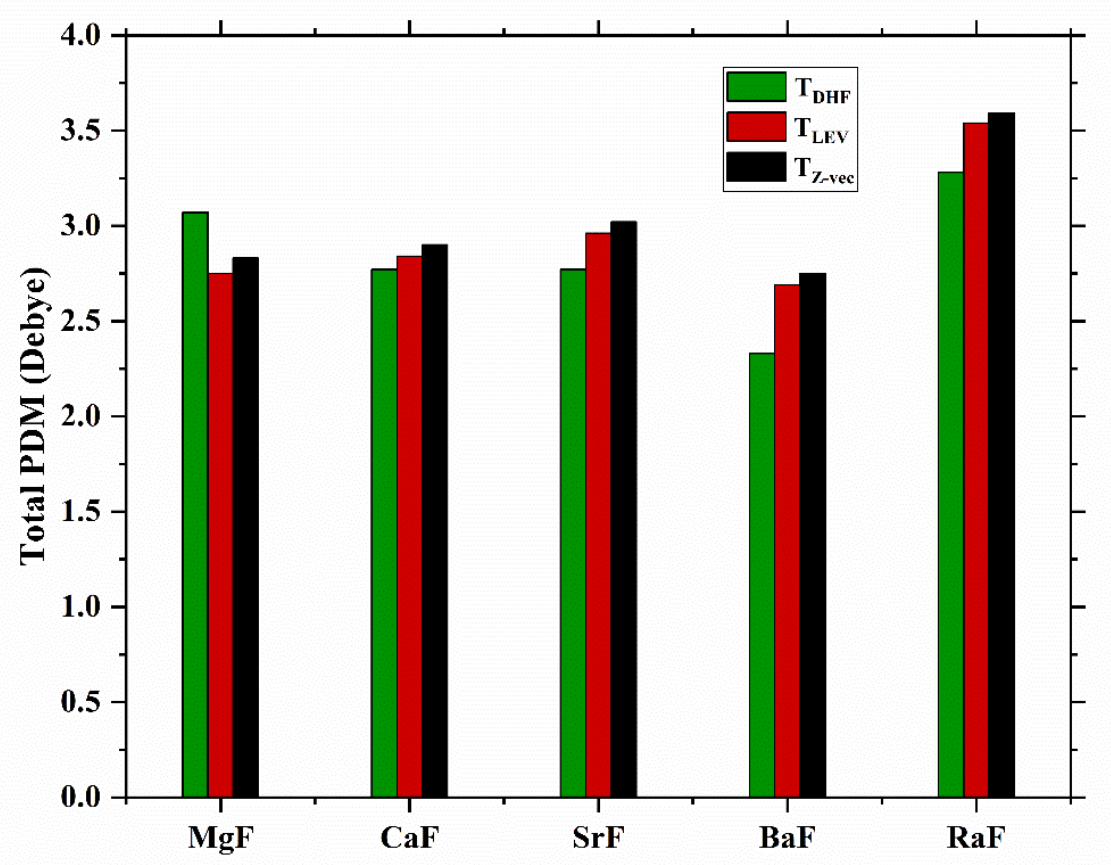

Fig 2: Total PDMs (in Debye) of AEMFs obtained from DHF ( $\left.\mathrm{T}_{\mathrm{DHF}}\right)$, LEV ( $\left.\mathrm{T}_{\mathrm{LEV}}\right)$ and Z-vector method $\left(\mathrm{T}_{\text {Z-vec }}\right)$

\section{IV.A.1. Comparison with available experimental values:}

The total PDMs obtained from our calculations show very good agreements with those obtained from other methods, previously reported by several groups. We shall not show an explicit comparison of our results with those of the previous works. In this paper our main concern is to compare the results obtained from LEV method and Z-vector method using same basis and correlation space. A detailed compilation of previously obtained results using several basis sets can be found in Ref. ${ }^{20} \&{ }^{19}$.

For the AEMF series, the experimental results were available only for $\mathrm{CaF}^{63}$ [3.07(7) $\left.\mathrm{D}\right], \mathrm{SrF}^{64}$ [3.4676(1) D] and $\mathrm{BaF}^{65}$ [3.179(3) D]. For these three molecules we compared the results obtained in our present work with the experimental ones. We present the deviation ( $\triangle \mathrm{PDM})$ of our calculated PDMs from the experimental results in Figure 3. It shows that if we consider only the $\mathrm{EC}_{\mathrm{DHF}}$ and $\mathrm{NC}$, neglecting the correlation corrections, then the PDMs are far away from the experimental results. The $\triangle \mathrm{PDM}$ becomes larger as we go from $\mathrm{CaF}$ to $\mathrm{SrF}$ to $\mathrm{BaF}$. This clearly indicates that electron correlation plays a crucial role in calculating the PDMs accurately, and one cannot really neglect this effect. Moreover, similar to the relativistic effect, the correlation contribution also becomes more pronounced as the molecule gets heavier. 
As can be seen from Figure 3, using larger basis leads to smaller deviation (DZ>TZ>QZ) when correlation is incorporated. This improvement in dipole moment with larger basis is expected as a greater number of basis functions (i.e. close to the completeness of basis set) can take care of correlation effects more accurately. However, when only $\mathrm{EC}_{\mathrm{DHF}}$ contribution is considered, $\mathrm{TZ}$ and $\mathrm{QZ}$ basis give larger deviations than the DZ basis. This discrepancy clearly indicates the lack of reliability on the average electron-electron interaction at the DHF level of theory, which misses out the proper electron correlation effect.

Both the LEV and Z-vector methods give much smaller errors in the total PDM values as compared to that obtained at the DHF level. It is worth mentioning here that higher order correlation and relativistic effects are not considered in our present calculations since it is beyond the scope of our present work. Due to this fact, the total PDMs might have some errors arising from it. Additionally, there could be some fortuitous cancellations of errors arising from different sources. However, it is to be noted that Z-vector method gives smaller error than the expectation value method for all the three molecules considered here, at both the DZ and TZ level of basis sets. The lower accuracy of the linear expectation value method can be attributed to the fact that it requires a linear approximation in the electronic contribution term (EC). Zvector method, on the other hand, does not suffer from any such forceful truncation. Therefore, the LEV method misses out some correlation and relaxation effects which are taken into account in the Z-vector method. As the basis set becomes as large as QZ, the two methods seem to give comparable errors. Thus, it can be inferred that at a relatively moderate size basis set like DZ or TZ, a combination of DHF and Zvector method produces more correct picture of the interplay between relativistic and correlation effect than that of the combination of DHF and Linear Expectation value method. Although the two methods give almost similar agreement with the experimental results in a saturated basis like QZ, such a large basis is not always computationally feasible to use for relativistic correlation calculations, especially for heavy molecules. 


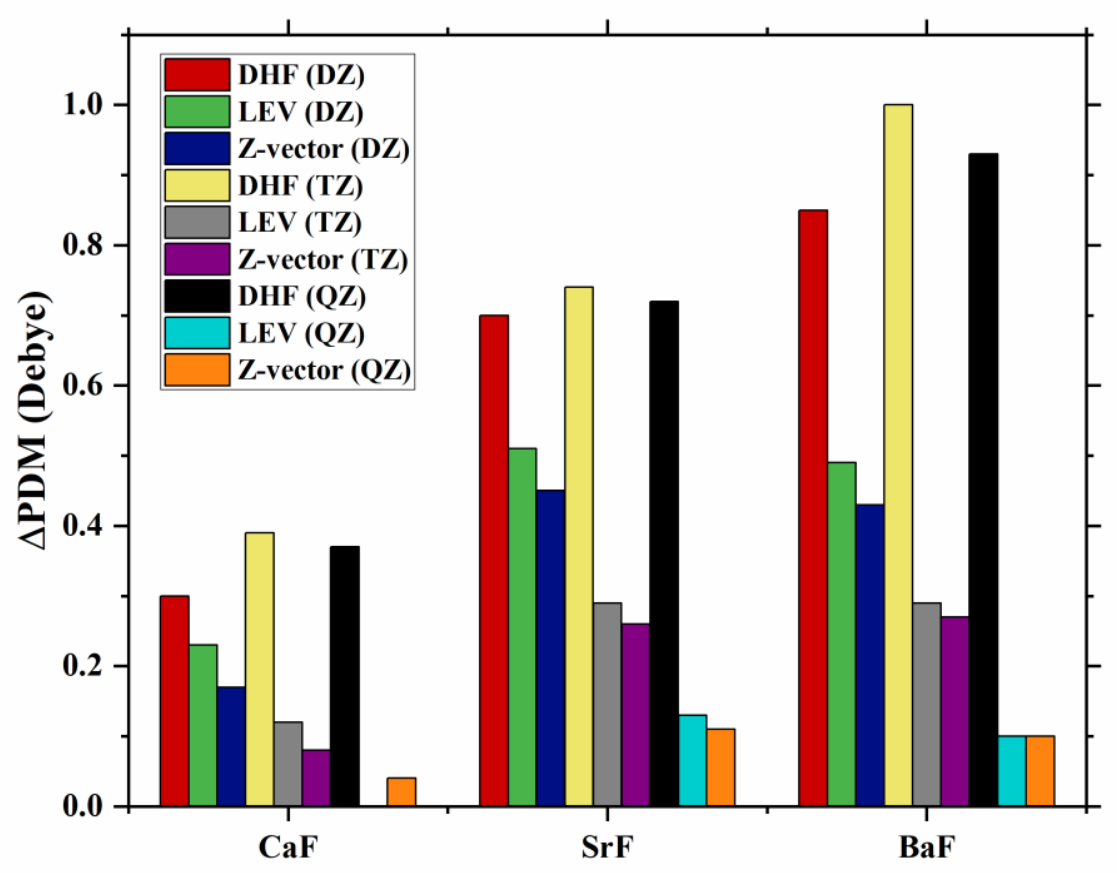

Fig 3: Errors in PDMs ( $\triangle \mathrm{PDM})$ in Debye, obtained from DHF, LEV and Z-vector method (using DZ, TZ and QZ basis sets), w.r.t the experimental PDMs

\section{IV.A.2. Effect of different relativistic treatments:}

Table 5: Different contributions to the PDM and the total PDMs (T) of SrF with TZ basis set (PDM is presented in Debye)

\begin{tabular}{cccccccc}
\hline Hamiltonian & NC & $\mathbf{E C}_{\mathbf{D H F}}$ & $\mathbf{E C}_{\text {corr }}$ & $\mathbf{E C}_{\text {corr }}$ & $\mathbf{T}_{\text {DHF }}$ & $\mathbf{T}_{\text {LEV }}$ & $\mathbf{T}_{\text {Z-vec }}$ \\
& & & $(\mathbf{L E V})$ & $(\mathbf{Z}$-vec $)$ & & & \\
\hline $\begin{array}{c}\text { Without Gaunt } \\
\text { 4C (TZ) }\end{array}$ & 89.70 & -92.43 & -0.45 & -0.48 & -2.73 & -3.18 & -3.21 \\
With Gaunt & 89.70 & -92.43 & -0.45 & -0.48 & -2.73 & -3.18 & -3.21 \\
4C (TZ) & & & & & & & \\
X2C (TZ) & 89.70 & -92.42 & -0.44 & -0.48 & -2.72 & -3.16 & -3.20 \\
\hline
\end{tabular}


As mentioned above we use only DC Hamiltonian to compute PDM of molecules. However, the higher order relativistic correction terms (Breit interaction) can have significant contribution to the molecular properties of heavy systems. To understand the role of relativistic effects in the calculation of the PDM, we have performed a TZ standard calculation using three different Hamiltonians- (i) exact two-component (X2C) relativistic Hamiltonian, (ii) fourcomponent (4C) Dirac-Coulomb (DC) Hamiltonian, and (iii) four component Dirac-CoulombGaunt (DCG) Hamiltonian for SrF which has the moderate size element in the AEMF series, as a test case. Table 5 represents the results obtained using the different Hamiltonians. We found that inclusion of the Gaunt term hardly changes the results for either of the two methods. This means, the higher order relativistic effects seem to have negligible contribution to molecular frame dipole moment of SrF. On the other hand, use of X2C Hamiltonian over the four component Hamiltonians leads to a decrease in magnitude of PDM by 0.01-0.02 Debye. Errors obtained from the 4C DC Hamiltonian and the X2C relativistic Hamiltonian have been shown in Figure 4.

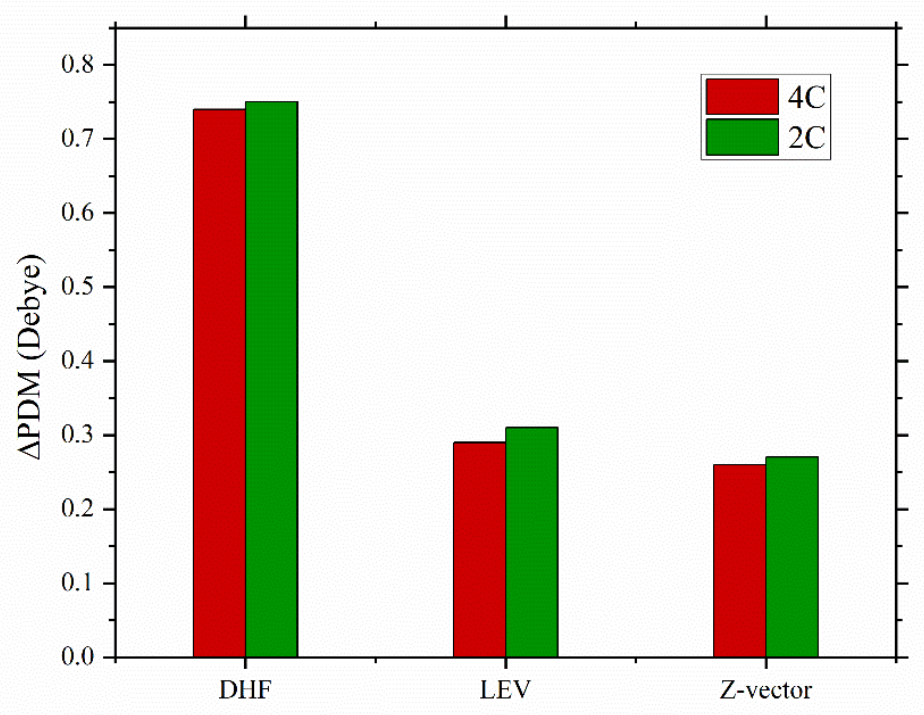

Fig 4: Errors in PDMs ( $\triangle \mathrm{PDM})$ of SrF, obtained from DHF, LEV and Z-vector method using fourcomponent and exact-two-component Hamiltonians (in TZ basis set), w.r.t the experimental PDMs

\section{IV.B. Group 2B Hydrides:}

For the group II-B-hydride series, the total PDMs and the contributions of different terms involved in the expression of the PDM, obtained from DZ and TZ quality basis sets are given in Table 6 and Table 7 , respectively. 
Table 6: Different contributions to the PDM and the total PDMs of group-IIB monohydrides with double zeta basis set. (PDM is presented in Debye)

\begin{tabular}{cccccccc}
\hline Molecule & $\mathbf{N C}$ & $\mathbf{E C}_{\mathbf{D H F}}$ & $\mathbf{E C}_{\text {corr }}$ & $\mathbf{E C}_{\text {corr }}$ & $\mathbf{T}_{\text {DHF }}$ & $\mathbf{T}_{\text {LEV }}$ & $\mathbf{T}_{\text {Z-vec }}$ \\
& & & $(\mathbf{L E V})$ & $(\mathbf{Z}$-vec $)$ & & & \\
\hline \hline $\mathrm{ZnH}$ & 7.66 & -8.43 & 0.37 & 0.31 & -0.77 & -0.40 & -0.46 \\
$\mathrm{CdH}$ & 8.55 & -9.60 & 0.55 & 0.44 & -1.05 & -0.50 & -0.61 \\
$\mathrm{HgH}$ & 8.48 & -9.10 & 0.56 & 0.45 & -0.62 & -0.06 & -0.17 \\
\hline
\end{tabular}

Table 7: Different contributions to the PDM and the total PDMs of group-IIB monohydrides with triple zeta basis set. (PDM is presented in Debye)

\begin{tabular}{cccccccc}
\hline Molecule & NC & $\mathbf{E C}_{\text {DHF }}$ & $\mathbf{E C}_{\text {corr }}$ & $\mathbf{E C}_{\text {corr }}$ & $\mathbf{T}_{\text {DHF }}$ & $\mathbf{T}_{\text {LEV }}$ & $\mathbf{T}_{\text {Z-vec }}$ \\
& & & $(\mathbf{L E V})$ & $(\mathbf{Z}$-vec $)$ & & & \\
\hline \hline $\mathrm{ZnH}$ & 7.66 & -8.45 & 0.29 & 0.22 & -0.79 & -0.50 & -0.57 \\
$\mathrm{CdH}$ & 8.55 & -9.63 & 0.45 & 0.34 & -1.08 & -0.63 & -0.74 \\
$\mathrm{HgH}$ & 8.48 & -9.10 & 0.49 & 0.37 & -0.62 & -0.13 & -0.25 \\
\hline
\end{tabular}

As we can see, for both the $\mathrm{DZ}$ and $\mathrm{TZ}$ quality basis sets, the nuclear contribution (NC) increases from $\mathrm{ZnH}$ to $\mathrm{CdH}$ while it decreases for $\mathrm{HgH}$. Exactly same trend is observed in the experimental bond length of the hydrides. It is the Lanthanide contraction that leads to a slight decrease in the bond length of $\mathrm{HgH}$ as compared to that of $\mathrm{CdH}$. $\mathrm{NC}$ increases linearly with the atomic number and equilibrium bond length of the molecule. Since we have chosen the metal centre as the origin, atomic number of $\mathrm{H}$ appears in the expression of $\mathrm{NC}$ term in equation (2) for all three molecules. Thus, in this case, the only determining factor is the equilibrium bond length which justifies the trend in $\mathrm{NC}$. The DHF contribution $\left(\mathrm{EC}_{\mathrm{DHF}}\right)$ also shows the same trend as the NC. Both LEV and Z-vector methods show a consistent increase in correlation contribution as the metal gets heavier.

Unlike the case of AEMF series, the group II-B monohydride series shows a similar trend in the total PDM, both with and without incorporating the correlation effect. In both the cases, the total PDM increases from $\mathrm{ZnH}$ to $\mathrm{CdH}$, but decreases for $\mathrm{HgH}$. This result can be explained considering the two determining factors behind the magnitude of the dipole moment: (i) difference in electronegativity between 
the two bonded atoms and (ii) distance between the charge separations. For $\mathrm{ZnH}$ and $\mathrm{CdH}$ these two factors act in opposite direction. Increasing bond length is the dominating factor over the decreasing electronegativity difference, and hence, on going from $\mathrm{ZnH}$ to $\mathrm{CdH}$, the dipole moment increases. On the other hand, on moving from $\mathrm{CdH}$ to $\mathrm{HgH}$ both the bond length and electronegativity difference decrease, thus resulting in a decrease in overall dipole moment.

Since the hydride series consistently gives smaller correlation contribution in Z-vector method than in the expectation value method, the magnitude of the total PDMs obtained from Z-vector method are always greater than those obtained from the expectation value method.

\section{IV.C. Contribution of Virtual Spinors and Core Correlation:}

Usually, in the calculation of molecular properties, higher energy virtual spinors are found to be important to obtain the proper picture of the correlation of inner-core electrons ${ }^{38,39}$. As mentioned earlier, we have considered the correlation of all electrons in our calculations of the PDMs of molecules. However, we have investigated the importance of the correlation of inner-core electrons, as well as the role of virtual spinors in the core-electron correlation, to calculate the molecular permanent dipole moment. For this purpose, $\mathrm{CdH}$ is chosen as the test case. We have performed two sets of calculations on $\mathrm{CdH}$ : in one case, all electrons and the occupied spinors (i.e. 49) are considered within the correlation space while in the other case, only the 19 outer valence electrons and the corresponding occupied spinors are taken into account, keeping the 30 inner core (1s-3d) spinors frozen, for the correlation calculation. In each set, we have discarded the high-lying virtual energy functions above three certain cutoff values (50, 500 and 1000 a.u.). A calculation with no virtual cutoff i.e. by taking all the virtual functions into consideration was also performed for each set. Basis set used for $\mathrm{Cd}$ is dyall.ae2z and for $\mathrm{H}$, cc-pCVDZ. Nuclear contribution (NC) and electronic contribution from DHF calculation $\left(\mathrm{EC}_{\mathrm{DHF}}\right)$, are found to be 8.554 and -9.597 Debye respectively.

We observe that increasing the correlation space by adding more and more virtual spinors does not change the total PDM at least up to three decimal places if the number of correlated electrons is kept constant. One should also note that freezing the inner core occupied spinors changes the correlation contribution by 0.013 and 0.01 Debye for the LEV and the Z-vector methods, respectively. This implies that high-lying virtual spinors seem to affect the total PDM of $\mathrm{CdH}$ very insignificantly, however, correlation of inner-core electrons has a small contribution to it, especially with the double zeta quality basis set. Figure 5 shows this fact clearly where the change in correlation contribution to the PDM (EC $\mathrm{Corr}_{\text {) }}$ has been presented as a function of the number of virtual spinors for $\mathrm{CdH}$, obtained from LEV method. 


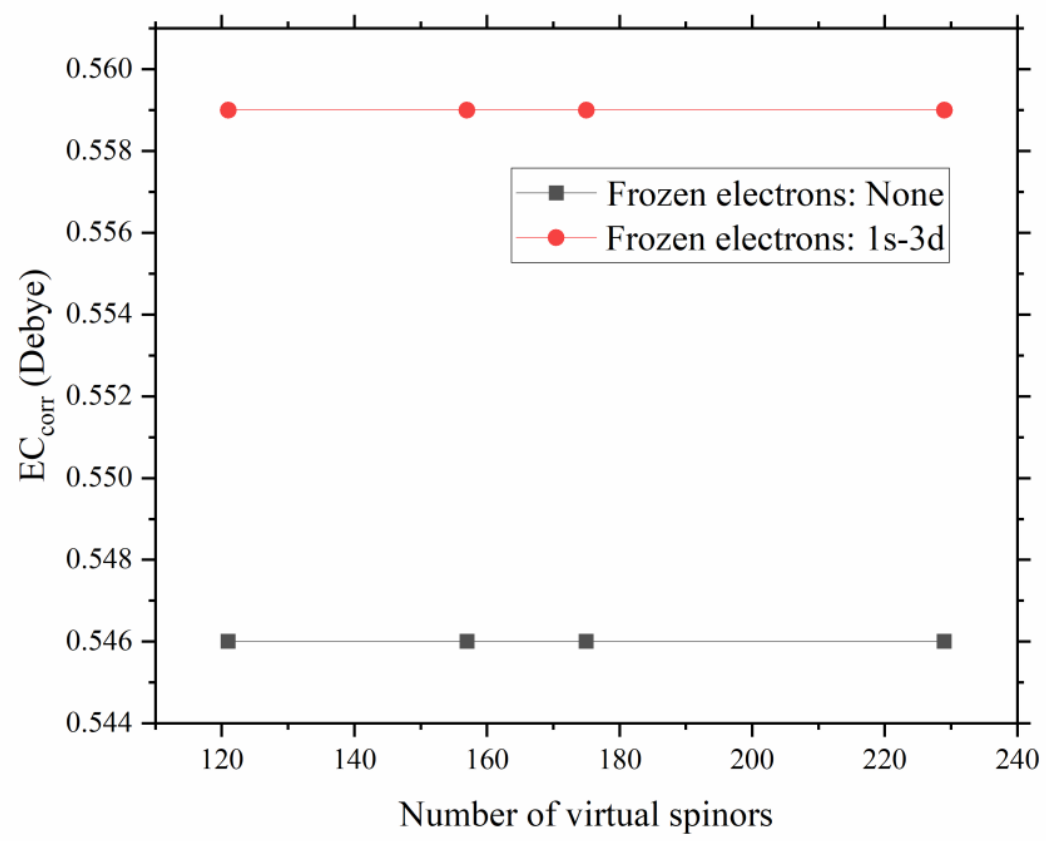

Fig 5: $\mathrm{EC}_{\text {corr }}$ (Debye) as a function of the number of virtual spinors for $\mathrm{CdH}$ obtained from linear expectation value method

\section{Conclusion:}

In this work, we have employed the Z-vector method which is a form of energy derivative approach, as well as the LEV method in the fully relativistic CC framework to calculate the molecular frame PDMs of the ground state of alkaline earth metal monofluorides and the monohydrides of group II-B elements. It is found that in DZ and TZ standard basis set, the Z-vector method gives significantly better result as compared to the LEV method in the RCC framework. As the size of the basis set increases, the difference in errors of these two methods decreases to some extent. Therefore, at least at a moderate size basis set, Zvector method would be a better choice than the linear expectation value method for accurate prediction of molecular PDM. We have also found that the correlation of inner-core electrons has a small influence on the PDMs of the studied molecules, whereas higher-energy virtual functions and the higher-order relativistic effects hardly affect this property.

\section{Acknowledgements:}

The authors thank SERB, India, for J. C. Bose Fellowship grant to Sourav Pal towards support of this work. Soumi Haldar thanks IIT Bombay for Senior Research Fellowship.

\section{References:}


1. C. Trefzger, C. Menotti, B. Capogrosso-Sansone and M. Lewenstein, J. Phys. B At. Mol. Opt. Phys., 2011, 44, 193001.

2. T. Mishra, R. V Pai, S. Ramanan, M. S. Luthra and B. P. Das, Phys. Rev. A, 2009, 80, 43614.

3. D. DeMille, Phys. Rev. Lett., 2002, 88, 67901.

4. $\quad$ D.-W. Wang, M. D. Lukin and E. Demler, Phys. Rev. Lett., 2006, 97, 180413.

5. A. C. Vutha, W. C. Campbell, Y. V Gurevich, N. R. Hutzler, M. Parsons, D. Patterson, E. Petrik, B. Spaun, J. M. Doyle, G. Gabrielse and others, J. Phys. B At. Mol. Opt. Phys., 2010, 43, 74007.

6. M. G. Kozlov and A. Derevianko, Phys. Rev. Lett., 2006, 97, 63001.

7. P. Sushkov and V. V Flarnbaurn, Zh. Eksp. Teor. Fiz, 1978, 75, 1208-1213.

8. T. Törring, W. E. Ernst and S. Kindt, J. Chem. Phys., 1984, 81, 4614-4619.

9. E. S. Rittner, J. Chem. Phys., 1951, 19, 1030-1035.

10. S. F. Rice, H. Martin and R. W. Field, J. Chem. Phys., 1985, 82, 5023-5034.

11. N. Honjou, G. F. Adams and D. R. Yarkony, J. Chem. Phys., 1983, 79, 4376-4381.

12. S. R. Langhoff, C. W. Bauschlicher Jr, H. Partridge and R. Ahlrichs, J. Chem. Phys., 1986, 84, 5025-5031.

13. J. Kobus, D. Moncrieff and S. Wilson, Phys. Rev. A, 2000, 62, 62503.

14. P. Bündgen, B. Engels and S. D. Peyerimhoff, Chem. Phys. Lett., 1991, 176, 407-412.

15. A. D. Buckingham and R. M. Olegário, Chem. Phys. Lett., 1993, 212, 253-259.

16. M. Abe, V. S. Prasannaa and B. P. Das, Phys. Rev. A, 2018, 97, 32515.

17. N. M. Fazil, V. S. Prasannaa, K. V. P. Latha, M. Abe and B. P. Das, Phys. Rev. A, 2018, 98, 32511.

18. V. S. Prasannaa, M. Abe and B. P. Das, Phys. Rev. A, 2014, 90, 52507.

19. V. S. Prasannaa, S. Sreerekha, M. Abe, V. M. Bannur and B. P. Das, Phys. Rev. A, 2016, 93, 42504.

20. R. Bala, H. S. Nataraj and M. K. Nayak, J. Phys. B At. Mol. Opt. Phys., 2019, 52, 85101.

21. L. Visscher, T. J. Lee and K. G. Dyall, J. Chem. Phys., 1996, 105, 8769-8776.

22. L. Visscher, K. G. Dyall and T. J. Lee, Int. J. Quantum Chem., 1995, 56, 411-419.

23. A. N. Petrov, N. S. Mosyagin, T. A. Isaev, A. V Titov, V. F. Ezhov, E. Eliav and U. Kaldor, Phys. Rev. Lett., 2002, 88, 73001.

24. E. Eliav, U. Kaldor and Y. Ishikawa, Phys. Rev. A, 1994, 49, 1724.

25. E. Eliav, U. Kaldor and Y. Ishikawa, Phys. Rev. A, 1994, 50, 1121.

26. E. Eliav, U. Kaldor and Y. Ishikawa, Phys. Rev. A, 1995, 51, 225.

27. A. Shee, L. Visscher and T. Saue, J. Chem. Phys., 2016, 145, 184107.

28. S. Sasmal, H. Pathak, M. K. Nayak, N. Vaval and S. Pal, Phys. Rev. A, 2015, 91, 22512. 
29. S. Sasmal, H. Pathak, M. K. Nayak, N. Vaval and S. Pal, Phys. Rev. A, 2015, 91, 30503.

30. S. Sasmal, H. Pathak, M. K. Nayak, N. Vaval and S. Pal, Phys. Rev. A, 2016, 93, 62506.

31. Y. Hao, L. F. Pašteka, L. Visscher, P. Aggarwal, H. L. Bethlem, A. Boeschoten, A. Borschevsky, M. Denis, K. Esajas, S. Hoekstra and others, J. Chem. Phys., 2019, 151, 34302.

32. V. S. Prasannaa, A. C. Vutha, M. Abe and B. P. Das, Phys. Rev. Lett., 2015, 114, 183001.

33. B. Jeziorski and R. Moszynski, Int. J. Quantum Chem., 1993, 48, 161-183.

34. J. Noga and M. Urban, Theor. Chim. Acta, 1988, 73, 291-306.

35. T. Korona and B. Jeziorski, J. Chem. Phys., 2006, 125, 184109.

36. T. Korona, Theor. Chem. Acc., 2011, 129, 15-30.

37. M. Abe, G. Gopakumar, M. Hada, B. P. Das, H. Tatewaki and D. Mukherjee, Phys. Rev. A, 2014, 90, 22501.

38. K. Talukdar, M. K. Nayak, N. Vaval and S. Pal, Phys. Rev. A, 2019, 99, 32503.

39. K. Talukdar, M. K. Nayak, N. Vaval and S. Pal, Phys. Rev. A, 2020, 101, 32505.

40. N. C. Handy and H. F. Schaefer III, J. Chem. Phys., 1984, 81, 5031-5033.

41. E. A. Salter, G. W. Trucks and R. J. Bartlett, J. Chem. Phys., 1989, 90, 1752-1766.

42. L. Visscher and K. G. Dyall, At. Data Nucl. Data Tables, 1997, 67, 207-224.

43. S. Pal, M. Durga Prasad and D. Mukherjee, Theor. Chim. Acta, 1983, 62, 523-536.

44. H. Koch, H. J. A. Jensen, P. Jo/rgensen, T. Helgaker, G. E. Scuseria and H. F. Schaefer III, J. Chem. Phys., 1990, 92, 4924-4940.

45. T. Helgaker, P. Jørgensen and N. C. Handy, Theor. Chim. Acta, 1989, 76, 227-245.

46. S. Pal, Phys. Rev. A, 1986, 34, 2682.

47. S. Pal, Phys. Rev. A, 1986, 33, 2240.

48. DIRAC, a relativistic ab initio electronic structure program, Release DIRAC17 (2017), written by L.Visscher, H. J. Aa. Jensen, R. Bast, and T. Saue, with contributions from V. Bakken, K. G. Dyall, S. Dubillard, U. Ekström, E. Eliav, T. Enevoldsen, E. Faßhauer, T. Fleig, O. Fossgaard, A. S. P. Gomes, E. D. Hedegård, T. Helgaker, J. Henriksson, M. Iliaš, Ch. R. Jacob, S. Knecht, S. Komorovský, O. Kullie, J. K. Lærdahl, C. V. Larsen, Y. S. Lee, H. S. Nataraj, M. K. Nayak, P. Norman, G. Olejniczak, J. Olsen, J. M. H. Olsen, Y. C. Park, J. K. Pedersen, M. Pernpointner, R. Di Remigio, K. Ruud, P. Sałek, B. Schimmelpfennig, A. Shee, J. Sikkema, A. J. Thorvaldsen, J. Thyssen, J. van Stralen, S. Villaume, O. Visser, T. Winther, and S. Yamamoto (see http://www.diracprogram.org).

49. A. Almoukhalalati, S. Knecht, H. J. A. Jensen, K. G. Dyall and T. Saue, J. Chem. Phys., 2016, $145,74104$.

50. K. G. Dyall, Theor. Chem. Acc., 2016, 135, 128. 
51. K. G. Dyall, J. Phys. Chem. A, 2009, 113, 12638-12644.

52. T. H. Dunning Jr, J. Chem. Phys., 1989, 90, 1007-1023.

53. K. G. Dyall, Theor. Chem. Acc., 2007, 117, 483-489.

54. K. G. Dyall, Theor. Chem. Acc., 2004, 112, 403-409.

55. K. G. Dyall, Theor. Chem. Acc., 2012, 131, 1217.

56. C. J. Cramer, Theor. Chem. Acc., 2008, 119, 523-524.

57. J. Emsley, Die Elemente, Walter de Gruyter, 2011.

58. https://cccbdb.nist.gov/

59. G. Herzberg and K. P. Huber, 1979.

60. A. D. Kudashov, A. N. Petrov, L. V Skripnikov, N. S. Mosyagin, T. A. Isaev, R. Berger and A. V Titov, Phys. Rev. A, 2014, 90, 52513.

61. L. B. Knight Jr and W. Weltner Jr, J. Chem. Phys., 1971, 55, 2061-2070.

62. J. W. Hovick and J. C. Poler, J. Chem. Educ., 2005, 82, 889.

63. W. J. Childs, L. S. Goodman, U. Nielsen and V. Pfeufer, J. Chem. Phys., 1984, 80, 2283-2287.

64. W. E. Ernst, J. Kändler, S. Kindt and T. Törring, Chem. Phys. Lett., 1985, 113, 351-354.

65. W. E. Ernst, J. Kändler and T. Törring, J. Chem. Phys., 1986, 84, 4769-4773. 